\title{
La Gestión de Calidad ante la Actual Dimensión Universitaria en España
}

\author{
Ángeles Cancela ${ }^{1}$, Ángel Sánchez ${ }^{1}$, Raquel Gandón ${ }^{2}$ y María J. Rey ${ }^{2}$ \\ (1) Dpto. de Ingeniería Química, Universidad de Vigo, 36310 Vigo-España \\ (e-mail: chiqui@uvigo.es; asanchez@uvigo.es) \\ (2) Área de Calidad, Universidad de Vigo, 36310 Vigo-España \\ (e-mail: areadecalidade@uvigo.es; areadecalidade01@uvigo.es)
}

\begin{abstract}
Resumen
Se describen y analizan las principales iniciativas para la garantía y mejora de la calidad que involucran a las universidades españolas, impulsadas fundamentalmente por el denominado Espacio Europeo de Educación Superior. A partir de un breve recorrido histórico del término "calidad", se establece el importante cambio, que aunque de forma lenta, ha ido penetrando en las Universidades Españolas, hasta alcanzar la dimensión actual. La calidad de las universidades ha dejado el segundo plano que ocupaba, para convertirse en el verdadero eje sobre el que gira toda su actividad socio-educativa. Se ofrece una visión general, de las acciones institucionales y estratégicas que en términos de calidad están asumiendo las universidades españolas, discutiendo los principales retos que todavía quedan por conseguir.
\end{abstract}

Palabras clave: calidad, garantía de calidad, certificación de calidad, planes de estudios, indicadores.

\section{The Qualit Management before the Current University Dimension in Spain}

\begin{abstract}
The main initiatives implemented by the Spanish universities in the context of the European Space for Higher Education, to improve and guarantee the quality of their work are described and analyzed. Starting from a brief historical description of the term "quality", the important changes, although relatively slow, that the Spanish universities have experienced to reach the current level, are established and discussed. Quality in the universities has left the background status that occupied in the past top become the real axis on which all educational activities are developed. A general vision of the institutional and strategic actions, that in quality terms are assuming the Spanish universities, is offered, discussing the main challenges that still need to be face.
\end{abstract}

Keywords: quality, quality assurance, quality certification, university curricula, indicators 


\section{INTRODUCCIÓN}

La Real Academia Española de la Lengua (RAE, 2010) define el término Calidad en sus dos primeras acepciones como: "propiedad o conjunto de propiedades inherentes a algo, que permiten juzgar su valor" y como "Superioridad o excelencia". Dicho término proviene del latín Qualitas que se refiere al Qué (Qualis) de un objeto. Otro de los aspectos más destacables del término calidad, es su progresivo aumento en el discurso empresarial e institucional. En una sociedad en la que existe una amplia oferta, la Calidad ha dejado de ser una ventaja competitiva para convertirse en una exigencia del mercado. Estos aspectos de fuerte carácter socioeconómico han penetrado en las instituciones educativas en general y en la Universidad en concreto impulsando acciones cuyos objetivos fundamentales pueden resumirse en: (i) eficacia y eficiencia en la gestión de recursos; (ii) rendición de cuentas a los grupos sociales implicados; (iii) competitividad nacional e internacional; y (iv) satisfacción de los grupos de interés.

En este contexto, la garantía de calidad puede describirse como la atención sistemática, estructurada y continua a la calidad en términos de su mantenimiento y mejora. "Son todas aquellas actividades encaminadas a asegurar la calidad tanto interna como externa". En el documento "Criterios y Directrices para la Garantía de Calidad en el Espacio Europeo de Educación Superior", desarrollado por la European Network for Quality Assurance in Higher Education (ENQA, 2005) se recoge que en el marco de las políticas y procesos formativos que se desarrollan en las Universidades, la garantía de la calidad ha de permitir a estas instituciones, demostrar que toman en serio la calidad de sus programas y títulos y que se comprometen a poner en marcha los medios que aseguren y demuestren esa calidad.

En los últimos años, la cultura de calidad ha ido calando poco a poco en la comunidad universitaria. Por vez primera en España, es legalmente exigible (R.D.1393/2007) que todos los Títulos Universitarios Oficiales estén sustentados por un Sistema de Garantía de Calidad, sin el cual no es posible la verificación y acreditación del mismo. Los procesos de Garantía de Calidad han ido trazando una línea ascendente, ganando así relevancia hasta convertirse en un aspecto con entidad propia pero inseparable del propio título. El concepto "Calidad" está en constante evolución, en continua adaptación a las necesidades de los grupos sociales. Así lo demuestran los análisis históricos realizados al respecto. La mayoría de autores, entre ellos Baeza (1999) distinguen seis etapas en el desarrollo y evolución de la calidad:

Primera etapa, trabajo artesanal: la única organización del trabajo existente durante siglos ha sido la organización por oficios, es decir, a través de personas expertas en todas las actividades que conformaban la producción. La industria se fundamentaba en los gremios y la artesanía y consecuentemente en la baja producción, la calidad se reduce a hacer las "cosas bien". La Universidad medieval, era una institución con una fuerte carga religiosa. El gremio de maestros universitarios (universitas magistrorum), se reservaba el derecho de admisión de aprendices y la graduación de los mismos, se supeditaba a la aprobación de pontífices y reyes (Sáinz y González, 1957).

Segunda etapa, pequeño empresario: esta etapa se caracteriza por la división del trabajo en tareas simples realizadas por personas de una capacidad mínima, de manera que cada persona realiza solo una pequeña parte del proceso, lo que origina que solo el nivel más alto de la Organización disponga de una visión global de todo el proceso. No se enseñaban los oficios sino una pequeña parte del trabajo. Esto originó aumentos drásticos en la producción dando lugar a la Revolución Industrial. Con ella, se produce un giro importante. La calidad, se centra en obtener la máxima producción para la obtención de beneficios. La Universidad Española de entonces, realiza intentos para aplicar un modelo uniforme a todas las Universidades de la Monarquía sobre todos a través del denominado Plan Caballero desarrollado a partir del año 1807 y se refuerza la figura del Rector. Se desarrolla la financiación a partir de los derechos de matrícula y académicos junto con los presupuestos del Estado. El resultado es el asentamiento de la Universidad Liberal.

Tercera etapa, postguerra mundial: Se produce una división de gran trascendencia desde la óptica de la Calidad. Mientras en Japón se desarrolla la máxima de "hacer las cosas bien a la primera", minimizando costes, y preocupándose por la satisfacción del cliente, en el resto del mundo se dedican a producir cuanto más mejor. En este contexto, alrededor de las décadas 40-50, la 
Universidad se convierte en elitista quedando sujeta a un control político riguroso (Miranda y Romero, 2006). Es a partir de los años 50 cuando aparecen con fuerza los considerados líderes de la calidad (Deming, 1975; Crosby, 1995; Ishikawa, 1985; Juran, 1989) que a posteriori han sentado las bases de las concepciones actuales sobre la calidad.

Cuarta etapa, aseguramiento de la calidad: A finales de los años 60 surge una nueva etapa en la que es necesario documentar el cumplimiento de la calidad. El aseguramiento de la calidad consiste fundamentalmente en un conjunto de actividades planeadas y sistemáticas, que son necesarias para proveer una confianza adecuada de que el producto o servicio va a satisfacer los requisitos de la calidad especificados. (Zúñiga, 1999).

Quinta etapa, calidad total: En los 80, se centra la atención en la calidad de los productos y el servicio al cliente. De esta forma, el cliente se convierte en el eje del sistema, entendiéndose la calidad como un factor estratégico de la empresa. La Calidad Total es definida como una forma de gestión de una organización basada en la participación de todos sus miembros y que pretende un éxito a largo plazo mediante la satisfacción del cliente y beneficios para todos los miembros de la organización y para la sociedad.

Sexta etapa, gestión de calidad: Por último la gestión de la calidad total, orientada hacia la excelencia empresarial. Ésta apunta más allá de la calidad de los productos y de la eficiencia de los procesos para fijarse en la organización en su globalidad. En Europa aparecen las normas ISO 9000 (UNE-EN ISO 9000, 2005) y el modelo europeo EFQM (EFQM, 2008).

Los procesos de Gestión de Calidad han experimentado, por lo tanto un importante auge hasta adquirir un verdadero cuerpo de conocimiento con una fuerte carga tecnológica, que va desde "El Control Calidad", el "Aseguramiento de la Calidad", "La Calidad Total" y la "Gestión de Calidad". (Miranda y Romero, 2006). Si nos centramos en las entidades públicas y más concretamente en las Universidades Españolas, la gestión de calidad parece haber tomado el impulso definitivo. El artículo 31 de la Ley Orgánica 4/2007, del 12 de Abril, que modifica la Ley Orgánica 6/2001, del 21 de Diciembre de las Universidades (LOM-LOU, 2007), recoge que la promoción y garantía de calidad de las universidades españolas, en el ámbito nacional e internacional, es un fin esencial de la política universitaria. Recoge, además, la necesidad de establecer criterios de garantía que faciliten la evaluación, certificación y acreditación de títulos universitarios. El Real Decreto 1393/2007, (R.D. 1393, 2007) por el que se establece la ordenación de las enseñanzas universitarias oficiales y los mecanismos que permitirán integrar el sistema universitario español en el Espacio Europeo de Educación Superior (EEES), hace referencia en su preámbulo a la importancia de los Sistemas de Garantía de Calidad como parte de los nuevos planes de estudio y como base para una eficaz organización de las enseñanzas. Asimismo, la nueva organización de las enseñanzas universitarias, propuesta por el Ministerio de Educación y Ciencia incorpora la garantía de calidad como uno de los elementos básicos que un plan de estudios debe contemplar (MEC, 2006)

El Marco Europeo de Educación Superior, ha generado un importante debate en las Universidades, en los gobiernos, en las agencias de calidad y en general en la sociedad, en torno a la Calidad de los Sistemas de Educación Superior. Han transcurrido once años desde, la Declaración de Bolonia (Bologna Declaration, 1999) y todavía la excelencia en la Educación Superior sigue siendo una "prioridad para la próxima década" (Louvain, 2009). Son múltiples los factores que han ralentizado los mecanismos para la excelencia en las instituciones de educación superior pero quizá el más relevante, es la dificultad de llegar a un consenso sobre el propio concepto de calidad. El concepto de calidad en la educación superior es potencialmente difícil de definir, e incluso aunque todos tengamos una aproximación intuitiva de lo que significa, no será fácil articularla (Murias et al., 2008). El enfoque que parece haber calado en las Universidades Europeas y las Españolas en concreto, está muy relacionado con aspectos competitivos. El concepto de Calidad elegido, que depende de la eficacia con la que cada institución lleva a cabo cada una de sus actividades, tiene un impacto directo sobre la competitividad y la reputación, conceptos que son a la vez muy relacionados con el mercado (Murias et al., 2008). 


\section{SISTEMAS DE GARANTÍA DE CALIDAD EN EL CONTEXTO ESPAÑOL}

El RD 1393/2007 de 29 de Octubre en su anexo I, punto 9, (R.D. 1393/2007) establece las directrices generales para el diseño e implantación de los Sistemas de Garantía de Calidad constituyendo el referente básico sobre el cual éstos descansan. Según la citada normativa, la Memoria para la solicitud de verificación de títulos oficiales, debe hacer referencia al Sistema de Garantía de Calidad que regula dicho título, estableciendo que la información contenida en este apartado puede referirse tanto a un sistema propio para el título, como a un sistema general de la Universidad o del Centro responsable de las enseñanzas, aplicable al título. Establece además cinco apartados o directrices para el diseño e implantación de los Sistemas de Garantía de Calidad, que concretan los contenidos mínimos para el desarrollo de éste. Éstos hacen referencia a: (i) los responsables del sistema de garantía de la calidad del plan de estudios; (ii) los procedimientos de evaluación y mejora de la calidad de la enseñanza y el profesorado; (iii) los procedimientos para garantizar la calidad de las prácticas externas y los programas de movilidad; (iv) los procedimientos de análisis de la inserción laboral de los graduados y de la satisfacción con la formación recibida y (v) los procedimientos para el análisis de la satisfacción de los distintos colectivos implicados (estudiantes, personal académico y de administración y servicios, etc.), y de atención a las sugerencias o reclamaciones junto con los criterios específicos en el caso de extinción del título.

Todo ello ha llevado a las Universidades a vertebrar Sistemas de Garantía de Calidad que comparten una serie de directrices comunes, pero conservan una amplia autonomía, permitiendo los ajustes necesarios de acuerdo a las características, recursos, contexto y necesidades de cada una de ellas. Para cumplir con los nuevos retos, han surgido iniciativas de gran interés, como la constitución de grupos de trabajo interuniversitario. Dos ejemplos de estos grupos, que aprovechan las experiencias y sinergias para cumplir objetivos comunes, son el Grupo GYA (GYA, 2007) y el Grupo Norte (Grupo Norte, 2007).

El primero de ellos, se constituye en Julio de 2007 por las Unidades Técnicas de Calidad de las Universidades de Cádiz, Coruña, Murcia, Politécnica de Cataluña, Santiago de Compostela, Valencia y Vigo con los siguientes objetivos: (i) analizar conjuntamente los diferentes elementos que constituyen el programa AUDIT (ANECA, 2007); (ii) consensuar un modelo estándar de procedimientos y su documentación asociada que recoja las aportaciones de las diferentes visiones y perspectivas de las Universidades participantes en el grupo; y (iii) dar a conocer las conclusiones y el trabajo a toda la comunidad universitaria interesada a través de una herramienta que pretende ser dinámica en tanto que está abierta a sugerencias, propuestas y aportaciones que contribuyan a la mejora de los procesos analizados.

El Grupo Norte formado por las Unidades Técnicas de Calidad de las Universidades de: Burgos, Cantabria, La Rioja, País Vasco, Pública de Navarra, Valladolid y Zaragoza, se crea con el objetivo de facilitar a las Universidades la implantación de los Sistemas de Garantía de Calidad. Tal y como se estable en su propia Web (http://www.unizar.es/unidad_calidad/procedimientos/index.htm), el grupo se fijó como objetivos generales colaborar en cuantas cuestiones afecten a la gestión de la calidad en sus universidades. A tal fin, se celebran reuniones periódicas de sus miembros y se facilitan el trabajo común de las Unidades Técnicas de Calidad, mediante la puesta en común de experiencias y buenas prácticas, utilizando un servidor común.

Las exigencias derivadas del EEES, obligan a las Universidades a vertebrar acciones ordenadas y sistemáticas de mejora continua, pero no se encuentran "solas" en este proceso, sino que el propio marco normativo atribuye a las agencias de calidad un papel fundamental. En el caso español, la LOU (LOU, 2001) establece en su artículo 31 que las funciones de evaluación y conducentes a la certificación y acreditación, corresponden a la Agencia Nacional de Evaluación de la Calidad y Acreditación y a los órganos de evaluación que la Ley de las Comunidades Autónomas determinen. Asimismo dedica el Artículo 32 a la Agencia Nacional de Evaluación de la Calidad y Acreditación (ANECA, 2002) estableciendo que se autoriza la creación de la Agencia Nacional de Evaluación de la Calidad y Acreditación, de acuerdo con las previsiones de la Ley de 
Agencias Estatales para la mejora de los servicios públicos (LEY 28/2006), a la que corresponden las funciones que le atribuye la presente Ley y la de elevar informes al ministerio competente en materia de Universidades y al Consejo de Universidades sobre el desarrollo de los procesos de evaluación, certificación y acreditación en España, a cuyos efectos podrá solicitar y prestar colaboración a los órganos de evaluación que, en su caso, existan en las Comunidades Autónomas.

Entre los órganos de evaluación de las Comunidades Autónomas del Estado Español, es importante citar a: la Agència per la Qualitat del Sistema Universitari de Catalunya, (AQU Catalunya, 2003), la Agencia para la Calidad del Sistema Universitario de Galicia (ACSUG, 2001), la Agencia Andaluza de Evaluación (AGAE, 2005), L'Agència de Qualitat Universitària de les Illes Balears (AQUIB, 2002) y otras hasta un total de once, que velan, en el ámbito de sus respectivas competencias, por el desarrollo de iniciativas para la mejora de la calidad de las Universidades, a través de las funciones de evaluación, certificación y acreditación previstas en la LOU. En este marco de apoyo a las universidades, algunas de las citadas agencias desarrollaron el Programa FIDES-AUDIT (ACSUG, 2007), que suma en la actualidad su tercera convocatoria. Este programa que se desarrolla de forma coordinada entre ANECA, ACSUC y AQU Catalunya, tiene como objetivo principal, guiar los procesos conducentes al diseño e implantación de Sistemas de Garantía de Calidad de los Centros Universitarios para afrontar la verificación y acreditación de títulos.

\section{DESARROLLO DE UN SGIC SEGÚN EL PROGRAMA FIDES-AUDIT}

De acuerdo a lo establecido en el Programa FIDES-AUDIT, los Sistemas de Garantía de Calidad de las Universidades, se desarrollan en cuatro fases: diseño, evaluación, implantación y certificación, las cuales se detallan a continuación:

\section{Fase de Diseño}

El diseño de los Sistemas de Garantía de Calidad (SGIC), comprende un conjunto de acciones sistemáticas que suponen cronológicamente: asumir el compromiso, planificar las acciones, realizar un diagnóstico y finalmente concretar la documentación. Supone que los centros en general y sus equipos de gobierno, en particular, formalicen su implicación de acuerdo a un cronograma previamente establecido, promuevan o lideren la implicación de sus grupos de interés y configuren la estructura organizativa adecuada, constituyendo y/o nombrando a los órganos responsables en materia de calidad. La planificación, abarca un conjunto de acciones destinadas a rentabilizar los tiempos, evitando el exceso de trabajo y/o la descoordinación y garantizando la conclusión de la etapa en las fechas previstas. La etapa de diagnóstico, implica la realización de un análisis del estado actual del centro, de sus resultados, necesidades, debilidades, fortalezas,...etc. Constituye el punto de inicio para el establecimiento claro y realista de los objetivos de calidad perseguidos y se concreta con el desarrollo de la totalidad de la documentación que configura el Sistema de Garantía de Calidad.

\section{Fase de Evaluación}

Una vez los centros han definido sus Sistemas de Garantía de Calidad, han de ser evaluados por entidades competentes que en el marco de los Programas Institucionales de Calidad son las Agencias de Calidad, que a través de sus Comisiones de Evaluación, emitirán un informe que podrá ser: (i) positivo, el Sistema de Garantía correspondiente puede ser implantado sin la obligación de introducir ninguna modificación en cuanto a su diseño; (ii) positivo condicionado, el Sistema de Garantía presentado puede ser implantado si se introducen las mejoras establecidas o (iii) negativo, el Sistema de Garantía de Calidad propuesto ha de ser reestructurado y deberá participar en la convocatoria siguiente.

\section{Fase de Implantación}

La fase de implantación, es probablemente, la que presenta una mayor complejidad para los centros, porque supone conjugar acciones de planificación, recogida de datos, formalización de los modos de actuar de acuerdo a los procedimientos establecidos, seguimiento, evaluación de resultados y objetivos, información pública y toma de decisiones para la mejora continua. 


\section{Fase de Certificación}

Supone la culminación del Sistema de Garantía de Calidad, aunque no su conclusión, puesto que se trata de un proceso cíclico, en constante evaluación y mejora. Cuarenta y cuatro de las Universidades Españolas, participantes en el Programa FIDES-AUDIT han recibido la certificación positiva de los Sistemas de Garantía de Calidad presentados en su primera convocatoria, lo que refleja la envergadura institucional del citado programa. El debate parece centrarse ahora en el progreso y resultados de las acciones emprendidas. Se ha dado un primer paso, en las Universidades Españolas consistente en la definición de Sistemas de Garantía de Calidad, falta ahora comprobar la continuidad y evolución de las acciones desde una dimensión fundamentalmente teórica a una dimensión práctica, en base a criterios de utilidad, eficacia y eficiencia. Conviene resaltar la enorme influencia que la Universidad recibe de la dimensión política, sometiéndose siempre a las dialécticas de ésta, lo que dificulta la configuración de un marco estable, también en lo que a la dimensión de la Calidad y/o excelencia se refiere.

\section{LOS PRINCIPALES RETOS DE LAS UNIVERSIDADES ESPAÑOLAS}

Aunque son importantes las iniciativas actuales que han impulsado el desarrollo de Sistemas de Garantía de Calidad en el contexto universitario español, lo cierto es, que no alcanza las cotas de progreso que imperan en el mundo empresarial. Aunque el contexto universitario y el empresarial son radicalmente distintos, éste último ofrece un marco de referencia que es indispensable tener en cuenta, para afrontar los retos que las instituciones universitarias europeas, en general y las españolas en particular, deben afrontar para contribuir al desarrollo de las comunidades en las que se asientan. Entre estos retos es importante destacar: (i) el establecimiento de un eficaz sistema de indicadores, que facilite la comparabilidad de los sistemas universitarios y la toma de decisiones para la mejora continua y solucione uno de los grandes problemas en la medición de la calidad de las Universidades que es la falta de consenso a la hora de definir los distintos tipos de indicadores. (Tobón et al., 2006); (ii) la mejora de la calidad del aprendizaje universitario, colocando al alumno en el centro del proceso y no como un mero receptor de la actividad docente (Biggs, 2005); (iii) el desarrollo de una universidad capaz de crear las sinergias sociales adecuadas para la mejora de la calidad de vida, convirtiéndose en una empresa del conocimiento, que asimila de forma crítica los mecanismos que regulan el mercado de los bienes y servicios (Malagón, 2005).

El reto es recuperar la Universidad Europea como motor de la sociedad y como referente social. Europa fue, durante siglos, lo que sus universidades afloraron de nuevos conocimientos y lo que los universitarios transfundieron a la sociedad, marcando un estilo de vida en el que el saber y el saber hacer eran la pauta distintiva entre países y naciones. La Universidad debe revitalizarse no sólo como formadora de los futuros titulados, sino también como impulsora de las transformaciones económicas y sociales. Debe cubrir sus finalidades formativas con la mayor calidad, pero también adoptar un papel activo en la difusión social del conocimiento y prefigurar la formación de los titulados que han de hacer realidad la Europa competitiva y solidaria del futuro. (Melle, 2005).

\section{CONCLUSIONES}

De los argumentos presentados, de su discusión y de los antecedentes de la literatura expuestos a través del artículo, se pueden obtener las siguientes conclusiones generales:

1.- el Marco Europeo de Educación Superior y las normas de desarrollo del mismo han supuesto un importante impulso en el desarrollo de iniciativas para la garantía y mejora de la calidad de las Universidades;

2.-el cumplimiento de las distintas directrices, ha llevado a las universidades a buscar fórmulas de colaboración que les permitan afrontar con éxito las acciones emprendidas; 
3.-las Agencias de Calidad cobran un protagonismo especial, desarrollando programas cuyo objetivo final es el apoyo a las Universidades, para el diseño e implantación de Sistemas de Garantía de Calidad;

4.-la nueva dimensión de la Calidad Universitaria pretende detectar las debilidades de las universidades, de sus centros y títulos con la finalidad de afrontar con éxito los retos que plantea la sociedad actual.

5.-en definitiva, la Calidad en las Universidades, ha dado un importante paso adelante en España, a través de medidas normativas fundamentalmente. Se abre, no obstante, un horizonte de incertidumbre acerca del alcance que ésta va adquirir. La influencia de las políticas generales en la Universidad Española ha impedido asentar hasta el momento, un marco de estabilidad propicio para el desarrollo amplio y práctico de la gestión de calidad en la misma.

\section{REFERENCIAS}

ACSUG, Agencia para la Calidad del Sistema Universitario de Galicia. Programa FIDES-AUDIT. http://www.acsug.es/galego/webs/fides.php. Acceso: 21 de Febrero (2010).

AGAE, Agencia Andaluza de Evaluación. http://www.acsug.es/galego/webs/fides.php. Acceso: 27 de Febrero (2010).

ANECA, Agencia Nacional de Evaluación de la Calidad y Acreditación (en línea), 2007. Programa AUDIT. http://www.aneca.es/actividadesevaluacion/evaluacionenseñanzas/audit.aspx. Acceso: 21 de Febrero (2010).

AQU Catalunya, Agéncia per a la Qualitat del Sistema Universitari de Catalunya. Programa FIDES-AUDIT. http://www.aqu.cat/ Acceso: 21 de Febrero (2010).

AQUIB, Agéncia de Qualitat Universitária de les Illes Balears http://www.aquib.org/cas/aquib/ Acceso: 14 de Marzo (2010).

Baeza, R., Educación Superior del Siglo XXI: Modelos para una gestión de calidad. Universidad del Mar, Chile (1999).

Biggs, J. B., Calidad del aprendizaje universitario, Narcea, Madrid, España (2005).

Bologna Declaration (en línea), 1999. http://www.bologna-

bergen2005.no/Docs/00Main_doc/990719BOLOGNA_DECLARATION.PDF. Acceso: 4 de Marzo (2010).

Crosby B. P., Phillip Crosby Associates (en línea), 2010. http://www.philipcrosby.com/pca/index.html. Acceso: 2 de Marzo (2010).

Deming. W.E. Calidad, productividad y competitividad. La salida de la crisis. Díaz de Santos. Madrid, España (1989).

EFQM, European Foundation for Quality Management (2008).

ENQA, European Association for Quality Assurance in Higher Education (en línea), 2004. Criterios y Directrices para la Garantía de Calidad en el Espacio Europeo de Educación Superior, http://www.enqa.eu/files/ESG\%20version\%20ESP.pdf. Acceso: 12 de Marzo (2010).

GYA, Grupo de Garantía y Acreditación (en línea), 2007. http://www.grupogya.es/. Acceso: 2 de Febrero (2010)

Grupo Norte (en línea), 2007.http://www.unizar.es/unidad_calidad/procedimientos/index.htm Acceso: 2 de Febrero (2010)

Ishikawa. K., ¿Qué es el Control Total de Calidad. La modalidad Japonesa. Ed La Norma, Madrid, España (1985). 
Juran M.J., Juran en el liderazgo para la calidad. New York, USA. (1989).

LEY 28/2006, de 18 de Julio, de Agencias estatales para la mejora de los servicios públicos, (B.O.E. 19/07/2001), http://www.boe.es/boe/dias/2006/07/19/pdfs/A27124-27132.pdf. Acceso 13 de Marzo (2010).

LEY 6/2001, de 21 de Diciembre, de Universidades. (B.O.E. 24/12/2001), http://www.boe.es/boe/dias/2001/12/24/ modificada por la Ley Orgánica 4/2007, de 12 de abril, (B.O.E. 13/04/2007) http://www.boe.es/boe/dias/2007/04/13/ . Acceso: 16 de Febrero (2010)

Louvaine, Commmuniqué of the Conference of European Ministers Responsible for Higher Education, Leuven and Louvaine la-Neuve, (en línea), 2009.

http://www.ond.vlaanderen.be/hogeronderwijs/bologna/conference/documents/Leuven_Louvain-laNeuve_Communiqué_April_2009.pdf. Acceso: 16 de Abril (2010).

Malagón, P.L., Universidad y sociedad: pertinencia y educación superior. Ed. Magisterio, Bogotá, Colombia (2005).

MEC, Ministerio de Educación y Ciencia, La Organización de las Enseñanzas Universitarias en España (en línea), 2006. http://www.um.es/estrategico/ficheros-sin-editar/usados/secdoc/OEU_MEC.pdf.Acceso 23 de Marzo (2010)

Melle, M., Los Retos de la Universidad Española en el Marco Europeo Universitario. (en línea), 2005. http://www.revistasculturales.com/articulos/99/temas-para-el-debate/356/2/los-retos-de-launiversidad-espanola-en-el-marco-europeo-universitario.html. Acceso 15 de abril (2010).

Miranda, S. L. y Romero, A. L., La calidad, su evolución histórica y algunos conceptos y términos asociados (en línea), 2006. http://www.gestiopolis.com/administracion-estrategia/archivo/lacalidad-historia-conceptos-y-terminos-asociados.zip. Acceso: 12 de Febrero (2010).

Murias, P., De Miguel, J.C. y Rodríguez, D., A Composite Indicator for University Quality Assesment: The Case of Spanish Higher Education System, Soc Indic Res 129-146 (2008).

RAE, Real Academia Española de la Lengua (en línea), 1713. http://www.rae.es/rae.html. Acceso: 4 de Febrero (2010).

R.D 1393/2007, de 29 de Octubre por el que se establece la ordenación de las enseñanzas universitarias oficiales, (B.O.E. no 260 30/10/2007), http://www.boe.es/boe/dias/2007/10/30/pdfs/A44037-44048.pdf . España (2007).

Sáinz, C. M. y González, A., Historia de las universidades hispánicas. Origen y desarrollo desde su aparición a nuestros días, $1^{a}$ edición, Ed. La Norma, Madrid, España (1957).

Tobón, S. et al. Competencias, calidad y educación superior. Ed. Magisterio, Bogota, Colombia (2006).

UNE-EN ISO 9000:2005, Norma Internacional, Sistemas de Gestión de Calidad. Fundamentos y Vocabulario, AEN/CTN 66 (2005).

Zuñiga, H., Mejoramiento continuo de la calidad en la educación superior, Universidad del Mar, Chile (1999). 